\title{
Imaging in Drug Discovery and Early Clinical Trials
}

M. Rudin, ed.

Basel, Switzerland: Birkhäuser Verlag, 2005, 393 pages, \$249
In recent years, drug discovery has evolved into a distinct branch of science. The impact of genomics on biomedical research and the development of efficient technology platforms allowing for highly parallel compound synthesis and screening procedures have led to a major paradigm shift in drug discovery and development. Efficient tools for the selection and validation of drug targets at both the preclinical and the clinical levels are required.

Noninvasive imaging and, in particular, molecular imaging are becoming essential technologies to support drug discovery and development. Imaging provides structural, functional, metabolic, and molecular readouts that are being applied to characterize a disease phenotype (diagnosis), to elucidate the molecular mechanisms involved, to evaluate drug efficacy and safety, and to identify potential biomarkers of the mechanism of action, efficacy, and safety of the drug. Noninvasive imaging techniques constitute a bridge between preclinical and clinical drug evaluation. Information such as target expression, target function, pathway activities, or cell migration can meanwhile be studied in the intact organism using various imaging modalities with reasonable spatial and temporal resolution. It is obvious that aside from the already established relevance in medical diagnostics, modern imaging techniques will play a major role in the development of novel therapies.

Progress in Drug Research is a prestigious series of monographs that provides extensive expert-written reviews on highly topical areas in current pharmaceutical and pharmacologic research. Imaging in Drug Discovery and Early Clinical Trials, volume 62 in the series, describes the contribution of imaging modalities to the various stages of drug discovery and development, from early target validation to their use in clinical development programs.
The 10 chapters of the book are devoted to describing the drug discovery process as such; the various imaging modalities being used both clinically and preclinically; applications of imaging during the optimization of a lead compound, such as bioavailability and efficacy; and the drug safety evaluation. Chapter 9 describes imaging biomarkers, their development, their advantages, and the potential pitfalls associated with their use. Chapter 10 deals with the regulatory issues that have to be addressed in the translation of imaging tools from animals to humans.

This book provides a comprehensive overview of the role of imaging in drug discovery today and discusses major developments and trends with regard to both technologies and applications. It fulfills the intended purpose, which is to illustrate the role of noninvasive imaging in modern drug discovery and development. The layout of this monograph follows the drug discovery process from target selection/ validation to clinical drug evaluation and ultimately to approval by regulatory authorities.

The book is written clearly, is highly readable, and is printed on good-quality paper. The selected figures and tables are of high quality and illustrate each particular point. A bibliography containing the seminal literature about the topic is included at the end of each chapter. I believe that this book will be useful for academic radiologists, nuclear physicians, and imaging scientists. It will also be helpful to clinicians who develop or evaluate new drugs. It would be a useful addition to any medical research library.

E. Edmund Kim M.D. Anderson Cancer Center University of Texas Houston, Texas 